\title{
Proposing a social ecological approach to address disparities in occupational exposures and health for low-wage and minority workers employed in small businesses
}

\author{
Maia Ingram ${ }^{1} \cdot$ Ann Marie A. Wolf ${ }^{2} \cdot$ Nicolas I. López-Gálvez $^{1} \cdot$ Stephanie C. Griffin ${ }^{1} \cdot$ Paloma I. Beamer $^{1}$
}

Received: 3 September 2020 / Accepted: 12 March 2021 / Published online: 27 March 2021

(c) The Author(s), under exclusive licence to Springer Nature America, Inc. 2021, corrected publication 2021

\begin{abstract}
Occupational disease and injuries are the 8th leading cause of death in the United States. Low-wage and minority workers are more likely to work in hazardous industries and are thus at greater risk. Within the small business sector, in particular, the health of low-wage and minority workers is threatened by a multitude of complex and interrelated factors that increase their risk for injuries, death, and even chronic disease. The COVID-19 pandemic has amplified these concerns, as many low-wage and minority workers are essential workers, and many small businesses are reopening with little to no guidance. The article describes work-related health risks and reviews current research on occupational and social ecological approaches to improving the health of minority and low-wage workers primarily employed by small businesses. We propose a conceptual framework that integrates the social ecological model with the hierarchy of controls to address work-related health among low-wage and minority workers specifically in the small business sector. Community-based strategies are recommended to engage small business owners and workers in efforts to address their immediate needs, while building towards sustainable policy change over time. These strategies are of particular importance as small businesses reopen in the ongoing pandemic.
\end{abstract}

Keywords Workplace exposure $\cdot$ Vulnerable occupations $\cdot$ Vulnerable populations $\cdot$ Health studies $\cdot$ Disease $\cdot$ Personal exposure

\section{Introduction}

On December 29, 1970, then President Richard Nixon signed the Occupational Safety and Health Act assuring safe working conditions for American workers and establishing the Occupational Safety and Health Administration. Yet almost 50 years after this historic event, far too many workers in the United States are getting sick or dying because of their job. Although occupational disease and injuries are greatly under recognized and underreported,

These authors contributed equally: Maia Ingram, Ann Marie A. Wolf, Paloma I. Beamer

Maia Ingram

maiai@ arizona.edu

1 College of Public Health, University of Arizona, Tucson, AZ, USA

2 Sonora Environmental Research Institute, Inc., Tucson, AZ, USA even before the COVID-19 pandemic, they were the 8th leading cause of death annually in the U.S., just ahead of automobile crashes [1]. The estimated total annual costs of occupational disease and injuries is around $\$ 250$ billion, which is similar to the annual cost of cancer [2]. Excluding injuries, over 49,000 deaths per year are attributable to occupational disease, with a disproportionate burden faced by minorities and low-wage workers $[1,3]$. These rates likely underestimate the true burden of occupational disease, because there may be several decades between a workplace exposure and the subsequent development of a disease, such as cancer.

Overrepresentation of minority workers in the most hazardous jobs increases their risk of injury, despite the fact that occupational injuries and disease are, by definition, preventable. Many of these workers are employed by firms with $20-50$ workers, representing $48 \%$ of the U.S. worker population. These businesses employ a disproportionate number of low-wage workers, many of whom are Latinx. Approximately one in five of all Latinx workers earn minimum wage or less, and one in four low-wage workers 
are immigrant workers, the majority of whom are Latinx [4]. These populations are often marginalized and do not have access to resources that would increase safety and reduce exposures at work. These structures of inequity have only been amplified during the COVID-19 pandemic, leading to many of the disparities we have seen in transmission and severity of outcomes [5]. On the 50th anniversary of the Occupational Safety and Health Act and in the midst of a global pandemic, there is an urgent need for community-based methods that build local agency to respond to existing and emerging health threats confronting workers who conduct some of the most hazardous and least appreciated yet essential work in our society. In this paper, we explore integration of a social ecological approach into occupational health efforts to address health risks of the most vulnerable workers in small businesses.

\section{Social ecological considerations in the health of low- wage workers}

The health of low-wage workers in the U.S. is threatened by a multitude of complex and interrelated factors that stem both directly from the work environment and indirectly from social and economic factors that increase their risk for injuries, death, acute infections like COVID-19 and even chronic disease [6]. According to OSHA, efforts to protect workers' health should follow a hierarchy of controls, where preferred controls are those that rely the least on workers' decisions and behaviors. All too often this is not the practice, and emphasis in the workplace is on workers being responsible for recognizing the hazards and protecting themselves. Efforts to incorporate a health promotion perspective into the workplace, also run the risk of focusing on individual lifestyle behavior change rather than on the larger contextual determinants of health of low-wage workers [7]. The result of relying on workers and employers is all too evident in the inability of OSHA to ensure the worker safety from COVID-19 exposure in the current pandemic [8]. Furthermore, this lack of regulatory action and oversight by OSHA and other government agencies has contributed to COVID disparities because "essential workers" are more likely to be minority and working for employers that are marginalized from these systems. There is growing recognition of the need to develop a holistic approach that integrates workplace safety and worksite health promotion, especially since addressing these issues in isolation may be more counter-productive than effective $[9,10]$.

The social ecological model (SEM) is an approach to health promotion that recognizes that individual health is a product of multiple influences of family, work, community, and the broader political environment. Baron et al. have proposed application of a social ecological framework to address converging individual, social, economic, and political factors that impact worker health [11]. A major obstacle in moving this agenda forward may be the dearth of evidence regarding effective systems- level interventions to address worksite health that take into account the multiple determinants of health and disease [12]. There are few tested models, for example, that integrate occupational safety and health concerns with individual and community health promotion. This is of particular concern for minority and low-wage workers in small businesses who have less access to resources on the higher levels of the occupational ecological framework due to social, cultural, and economic barriers [11]. While our work has previously focused on reducing hazardous chemical exposures to these workers in small businesses, these are the same workers who have had to interact the most with the public during the COVID-19 pandemic with the least access to safety protocols, exposure mitigation strategies, and personal protective equipment (PPE).

The principal objective of this article is to propose a conceptual framework that integrates the SEM with the hierarchy of controls to address work-related exposures and injuries specifically in the small business sector. We begin with a description of work-related health risks and review current research on social ecological approaches to improving the health of Latinx and low-wage workers in small businesses. We then propose a model designed to integrate occupational health approaches with the SEM. Finally, we provide recommendations intended to advance the development of evidence-based strategies to address occupational health equity for low-wage workers, particularly in small businesses. Implementation of these recommendations during the COVID-19 pandemic could have significant impacts on reducing additional health disparities in this vulnerable population.

\section{Health risks to low wage workers in small business}

Small businesses are mostly concentrated in the professional services sector and include worksites that place workers at risk for chemical exposures and interactions with the general public, such as auto repair and beauty salons. Many use solvents and other products containing volatile organic compounds (VOCs) that are associated with asthma, cancer, Alzheimer's disease, encephalopathy, decreased memory ability, vision problems, peripheral neuropathy, loss of hearing or smell, and lowered semen quality [13-16]. Many businesses are using more chemical products to disinfect their workplaces in order to reduce exposure to the novel coronavirus leading to concerns of exposures related to improper use of disinfectants and lack of appropriate protection and ventilation [17]. Given that 
there are more workers in small businesses that are less likely to report occupational illnesses and injuries, it is impossible to know how the burden of occupational illness and injuries compared to larger businesses [18, 19]. Overall, workplace injury for Latinx is higher than any other ethnic group and Latinx workers are more likely to die on the job [20]. To the extent that income is indicative of health disparities, these workers also enjoy fewer years of life, have less access to health care and experience greater illness and disease than people in higher income brackets [21]. Greater risk of COVID-19 disease severity for Latinx people is in part explained by occupation or the occupation of household members, with Latinx workers less likely to be able to work remotely [22].

\section{Challenges to the practice of worksite safety in small business}

With respect to worksite safety and health, small businesses are less likely to employ industrial hygiene consultants even when using hazardous chemicals or to prevent infectious disease [23]. Many owners and employees lack language, literacy, and computer skills to access virtual resources [24]. There are also major issues deterring small businesses from trusting regulatory agencies or accessing other occupational health and safety resources. For Latinx-owned business, there is a lack of linguistically and culturally appropriate occupational safety and health resources. Online resources are often literal translations of existing English materials and of inconsistent quality [25]. Immigrant workers, who may have marginal economic and immigration status, distrust government agencies. These workers are not typically represented by labor unions and may fear losing their jobs if they question the hazards in their workplace. Small businesses also lack clear and consistent guidance from regulatory agencies on how to reduce coronavirus transmission, and many have had to develop protocols on their own in attempt to protect their workers and clients.

Despite substantial research on strategies to reduce workplace exposures and attempts by agencies to reach small businesses, small business owners and workers are generally unaware of their rights and responsibilities, and more importantly, of the hazards associated with the chemicals they use, including cleaning and disinfection products [26]. The lack of safety controls in small business results in improper handling and use of solvents and cleaning products coupled with inadequate use of PPE and engineering controls [27]. OSHA's hierarchy of controls is a widely accepted approach designed to limit exposures and protect workers' health. As illustrated in Fig. 1, at the bottom of the hierarchy, the use of PPE, such as gloves and respirators, relies upon individual behavior and is the most common, yet least effective approach. The hierarchy
Hierarchy of Controls

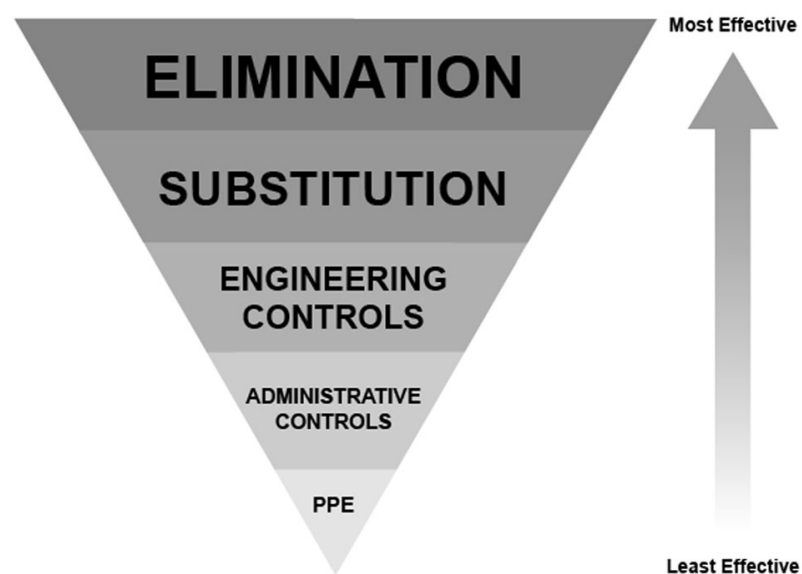

Fig. 1 Hierarchy of controls. The ascending arrow denotes the increasing effectiveness of exposure control strategies.

emphasizes the importance of substitution and elimination as higher-level controls that are both feasible and more effective to protect workers and the broader community from hazardous exposures. While the hierarchy offers a practical framework for addressing risk, it does not provide an approach to addressing the challenges inherent in the small business environment, nor to incorporating the larger socioeconomic and political context influencing work and community environmental circumstances [11]. The SEM recognizes underlying societal conditions of health behavior that can be mitigated through community involvement and public health policy. Integration of these two complementary models offers a potential approach for effective and sustainable changes in small business practices and ultimately community health.

\section{Relevance of the social ecological model to improving occupational health}

The COVID-19 pandemic underscores the importance of the SEM in moving beyond a health promotion focus on lifestyle change to include the social, economic, and political contexts that influence individual decisions and opportunities [28]. The SEM illustrates embedded levels of influence-individual, interpersonal, organizational, community, and public policy, which interact to impact health outcomes (Fig. 2). Recognizing the role of social and economic factors in creating and perpetuating health disparities, the SEM incorporates a response to individual needs while stressing incorporation of a systems approach that challenges entrenched structural barriers and inequities. The concentric circles that make up the SEM thus direct public health professionals to design strategies that address school, 


\section{Social Ecological Model}

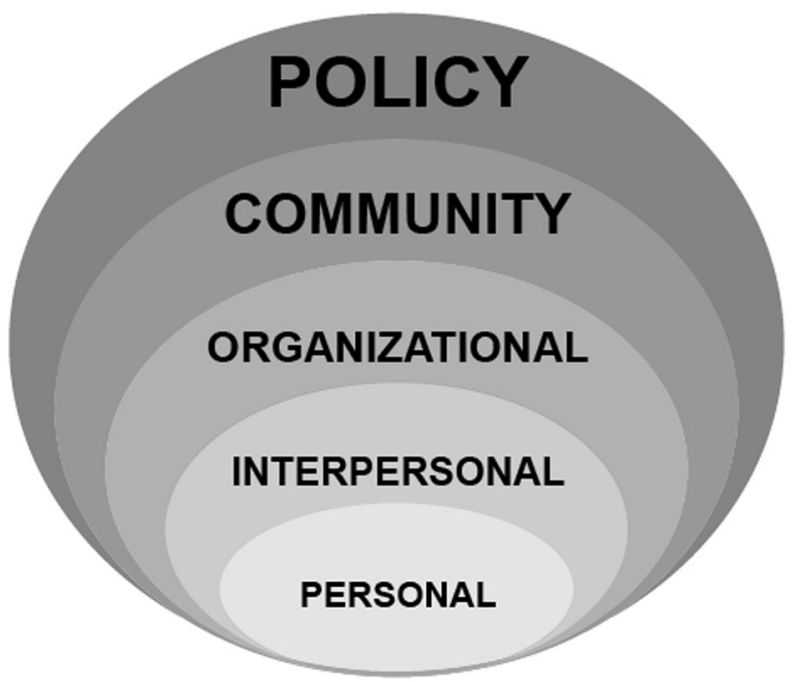

Fig. 2 Social ecological model. The concentric circles illustrate the interdependent and interactive influences on health.

work, and community conditions, many of which require altering public health policy on a worksite/organizational, community, or state/national level. Researchers recommend incorporating employee health into health promotion efforts [11], and the worksite has become a focal point of many health promotion strategies to address organizational context as part of a SEM approach [6, 29]. Unfortunately, recent systematic reviews demonstrate that few interventions that utilize the SEM incorporate multi-level strategies and fewer actually intervene at the public policy level [29]. These interventions tend to focus on the individual, interpersonal and organizational levels, for example pairing educational flyers with affordable food options in worksite cafeterias or creating a working environment that is more conducive to physical activity.

The intent of the ecological paradigm in targeting low-wage workers in the small business environment is to illuminate economic and social pressures that occur for both the worker and the small business owner. Economic stress may cause workers themselves to place themselves at greater risk by working long hours and missing meals in order to meet the financial needs of their families, and perhaps even work while being ill or in unsafe conditions such as during the COVID-19 pandemic. Workers in small businesses are more likely to be uninsured, not have paid time off, and less likely to seek preventive health services or health care when they experience symptoms of illness. Small business owners, many of whom are from the community and/or employ members of their family, may not be aware of the impact of the work environment on retention and productivity or have the infrastructure and human resources to support health protection and promotion efforts. A systematic review of health promotion programs addressing low-wage workers identified only a few that utilized the social ecological approach. The interventions targeted blue collar workers and sought to integrate administrative level changes to increase occupational health and safety with individual-level health promotion activities [10]. Primary outcomes focused on individual behavior change, and only one of four sites integrated the administrative health and safety protocol recommended by the intervention. Further, there is little evidence regarding the effectiveness of these or other health promotion programs in addressing health disparities [21].

A handful of occupational health studies employ the SEM to elucidate social, political, and economic factors in creating unsafe work environments. These studies do not all address workers in small businesses, but we include them to provide examples of how the SEM model can be applied in occupational health. A qualitative SEM study of cedar block cutters in Washington State found that the nonexistence of on-site personal protection equipment and safety regulations was compounded by lack of workers' compensation or health insurance on an organizational level, as well as undocumented status and racial discrimination as factors that converged to create precarious and unhealthy conditions for workers [30]. In another instance, community concern about air pollution caused by small businesses initiated a series of community-based efforts that involved community mapping, culturally relevant educational materials, small business training, and peer education visits [31]. Notably, these studies utilized a community-based participatory research (CBPR) approach that included building trust with both owners and workers, identifying priorities with research partners and moving research findings towards some type of action to address health disparities. The inclusion of community advisory boards or task forces in the CBPR studies can help move interventions toward policy solutions. A community task force developed as part of a CBPR intervention to address worker injury associated with hardwood floor finishing helped identify challenges in engaging the workers and the business owners and underscored the importance of the involvement of industry stakeholders in identifying effective strategies. The intervention also moved beyond efforts to improve adherence to personal protection recommendations and the voluntary substitution of flammable products by the business to include regulatory changes in Massachusetts to ban dangerous products [32].

In facing the COVID-19 pandemic, Eaton and Kalichman conducted a SEM analysis of factors influencing our capacity to collectively respond to the challenges we currently face [28]. Using the HIV epidemic as a case study, they point out that in the absence of clear and enforced 
national or even state policy, intrapersonal, interpersonal, community interventions can contribute to the desired outcome of disease eradication. Community mobilization efforts in the U.S. were ultimately highly effective at changing the federal response, further demonstrating the importance of the community and society in the SEM. With COVID-19 disproportionately affecting low-wage and often essential workers, the need for cohesive and sustained policy approaches that support small business during closure or to fund modifications to workspace are essential.

\section{Integrating SEM and the hierarchy of controls}

Graphic juxtaposition of the SEM with the hierarchy of controls illustrates the need to engage stakeholders at each level of influence to identify and address priorities relevant to employee safety and health (Fig. 3). While not exhaustive, the entities listed within each level of the SEM on Fig. 3 offer potential targets for interventions that applied together have greater potential to create sustainable change. Within the SEM, the hierarchy of controls is a tool that can be implemented at all SEM levels of intervention. For example, although PPE targets the individual, and is therefore least recommended, the SEM demonstrates that PPE can be promoted at the organizational level by the business owner as part of their administrative protocols. The higher level controls of elimination and substitution, occur at the organizational or small business level, but should be facilitated by public policy that encourages industry to create safer and less toxic chemicals. With respect to COVID-19, elimination may translate to reducing occupancy of workers and clients, and temporary policies to close certain types of small businesses that pose the greatest risk not only to the public but to their employees.

As Fig. 3 makes clear, there is a notable gap in exposure control strategies addressing the community domain of the SEM. There are, however, numerous stakeholders within

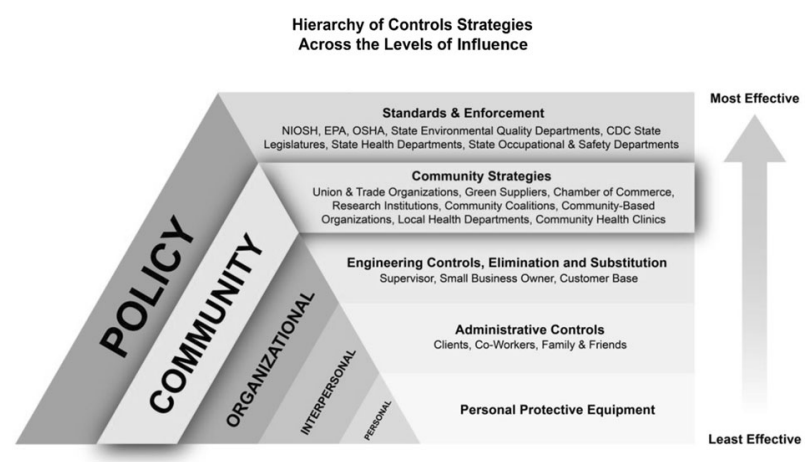

Fig. 3 Integration of SEM and HoC. Application of hierarchy of control strategies across the levels of the social ecological model. the community domain that can be targeted to create a holistic intervention that ensures that workers and businesses are identifying and addressing their immediate needs, while also building towards policy change that will sustain positive change over time. Local health departments, for example, have a mandate to conduct community assessments and develop programs and policies that address identified needs. The inclusion of small business owners and employees in these efforts would greatly expand their reach, as well as focus efforts to improve the influence of the workplace on the health of low-wage workers. Given the role that small businesses play in reducing and preventing COVID-19 transmission in the community, increased dialog between these stakeholders is essential to prevent both community and worker exposures and economic impacts on small businesses as we adjust to the "new normal". We propose community-based strategies designed to engage workers and businesses in administrative controls that address their immediate needs, while also building towards policy change that will sustain positive change over time. These strategies are described below with examples in Table 1.

\section{Community-based participatory research (CBPR)}

We propose CBPR as the foundation for engaging the small business community, made up of owners, contractors, workers, family members, and clients, in the process of describing relevant issues and identifying acceptable solutions within the work environment. There is a history of environmental justice research efforts that actively engage communities disproportionately affected by industrial exposure to identify threats and to mitigate the health burden [11]. When incorporated into a research approach, the CBPR core principles ensure that community members are partners in, rather than subjects of, research and that community priorities are at the forefront of research questions. When applied to small business context, the CBPR process elevates the experience of small business workers and employees, increasing the potential for acceptable, feasible, and viable solutions to be identified and put into practice.

\section{Community health worker workforce}

A proposed strategy for bridging the gap in the community domain and to bridge the gap between occupational health policy and small businesses and low-wage workers is to engage the community health worker (CHW) workforce. CHWs are front-line health workers with close and trusting relationships with community members and a long history of addressing health disparities. CHWs can be directly involved addressing the impact of COVID-19 in small business through community education, as well as in 
Table 1 Worksite health and safety interventions across the social ecological framework.

\begin{tabular}{|c|c|c|}
\hline Social ecological domain & Strategies & Examples \\
\hline Individual & $\begin{array}{l}\text { - Employee health considered holistically in terms of both } \\
\text { minimizing hazardous exposures and health promoting activities }\end{array}$ & $\begin{array}{l}\text { - Employer providing free PPE to workers. } \\
\text { - Work expectations consider limitations caused by PPE } \\
\text { - Employees encouraged not to work when ill }\end{array}$ \\
\hline Interpersonal & $\begin{array}{l}\text { - Customers oriented toward the potential product risk for worker, } \\
\text { client, and community } \\
\text { - Workers are encouraged to engage in walking groups. }\end{array}$ & $\begin{array}{l}\text { - In-service trainings on products and potential exposures } \\
\text { - Client customer contracts on product use } \\
\text { - Workers encouraged to support each other }\end{array}$ \\
\hline Organizational & $\begin{array}{l}\text { - Administrative safety protocols } \\
\text { - Engineering interventions } \\
\text { - Increased access to health care }\end{array}$ & $\begin{array}{l}\text { - CHW-facilitated worksite assessments } \\
\text { - Increased ventilation } \\
\text { - Workplace health screenings } \\
\text { - Employer negotiated health insurance }\end{array}$ \\
\hline Community & $\begin{array}{l}\text { - Community-based participatory research } \\
\text { - Community coalitions } \\
\text { - Community campaigns } \\
\text { - Building change agent capacity }\end{array}$ & $\begin{array}{l}\text { - Business owners and workers, family members and clients } \\
\text { engaged in documenting and prioritizing issues } \\
\text { - CHW ambassadors } \\
\text { - Promoting of best practices }\end{array}$ \\
\hline Policy & $\begin{array}{l}\text { - State and National Standards } \\
\text { - Worksite resources and enforcement }\end{array}$ & $\begin{array}{l}\text { - Ventilation standards and enforcement for nail shops } \\
\text { - Requirements for more detailed product labeling } \\
\text { - County funds for worksite improvements that benefit } \\
\text { community health }\end{array}$ \\
\hline
\end{tabular}

addressing the social and economic needs of workers. CHW interventions have contributed to increased access to healthcare, improved quality of care, compliance with prescribed care, reduced costs of care, and improved health status. CHWs link community members to governmental, educational, and social services that are otherwise inaccessible due to language and culture barriers. Involvement of CHWs in developing community-based interventions is also advantageous in addressing systems level changes to have broad and lasting effects on health outcomes. With occupational health research specifically, there are only a few tested CHW interventions that provide occupational health education to small businesses or address exposure reductions [31]. In the proposed model, CHWs can be ambassadors of both individual and business health promotion in constructively engaging small businesses and workers to reduce exposures in the work environment and to improve the overall wellbeing of the workforce. As local change agents, CHWs are also proactive in pursuing policy change, as well as engaging community members in advocating for change that can positively impact the health of the community. Thus, CHWs can potentially aid in improving occupational health in small businesses in their communities by working through all the domains of influence in our proposed model.

\section{Targeted actions}

Targeted actions are specific activities designed to culminate in a shift in health status in the community of focus. A pivotal activity is to build the capacity of change agents within the community, such as owners and employees, who can address environmental and/or policy change. Within the small business sector, a campaign to reduce the reliance on respirators to reduce VOC exposures could be augmented by financial incentives to install ventilation systems or other engineering controls that would in turn also reduce COVID19 transmission. Additionally, CHWs can integrate target actions to lower VOCs with health promotion efforts, such as increasing access to health care for workers in small businesses that generally do not offer health insurance.

\section{Community coalitions}

Community coalitions are often included in CBPR projects, both to enhance the integrity of the research and to increase the potential for action related to study findings [33]. Local health departments commonly rely upon community coalitions to address equity in their community health improvement plans. Community coalitions that include a broad array of stakeholders increase the potential that members will identify relevant issues and acceptable solutions, which can then be pursued through community mobilization and leveraging the political influence of partner organizations. In occupational health, coalitions may emerge in response to an environmental threat. For example, environmental justice coalitions, such as Adhikaar, have been working for several years to improve nail salons working conditions. Adhikaar partnered with the New York Committee for Occupational Safety and Health to make the New York Healthy Nail Salons Coalition to improve labor conditions in nail salons and to press for changes in future policies that can help with fair wages and safe working environments in nail salons [34]. While not expressly employing the SEM, efforts initiated at the worker, shop, and community level in NYC resulted in the adoption of a state-wide ventilation standard based on an application of the hierarchy of controls [35]. During the COVID-19 pandemic, coalitions of small business sectors may be some of the most effective mechanisms to share best practices for minimizing COVID exposure within a certain business 
type, ensuring that those practices are consistent across businesses in those sectors, and ultimately, leading towards reduction in community transmission through common practices and policies.

\section{Technical advisory committees (TACs)}

The role of a TAC is to augment decision-making processes with industry-related expertise. TACs have been useful in helping to integrate public health concerns into policy decisions. TACs gained traction in the 1990s when the U.S. Environmental Protection Agency began using them to increase community input in the development of remediation plans [36]. Many of U.S. EPA's workplace practices were developed by the U.S. EPA's Design for the Environment Program in partnership with small businesses, including shops and contractors, chemical and product manufacturers and distributors, trade associations, communities, and other federal, state, and local government agencies. Some of the best guidance for small businesses during the COVID-19 pandemic has come from these sorts of organizations. For example, the Automobile Service Association provided guidance in multiple languages for auto repair shops very early during the pandemic.

\section{Public health advocacy}

Public health policy is relevant to almost every aspect of daily life and can have major and sustainable impact health outcomes. Advocating for policies that can improve healthy environments is thus a critical component of an integrated approach to the health of low-wage workers in small businesses. In the proposed model, public health advocacy is a distinct strategy because of its importance in addressing health disparities; however, it is integral to all of the strategies presented in the model. For example, advocacy efforts can be rooted in the needs of the community, as identified through community-engaged research and community coalitions, as well supported by federal and state associations with technical expertise. While not related to small businesses, an example is the elimination of "el cortito", the short-handled hoe, an agricultural tool preferred by growers in California that caused musculoskeletal injury to farm workers. Farm workers successfully advocated for abolition of the tool under the leadership of Cesar Chavez [37]. At no other recent time in history has the importance of public health policy on workers' and the overall public's health been highlighted as during the COVID-19 pandemic, and coalitions of small businesses and their workers could advocate for their collective protection. As different states in the U.S. and different countries across the world have enacted different public health policies regarding small businesses, there have been dramatic implications on both community transmission and economic impacts to small businesses. Time will tell what the long-term consequences and or benefits are of those policies on different communities and the low-wage workers that serve them.

\section{Conclusion}

Minority-owned small business and low-wage workers face social and economic conditions that place them at high risk for occupation-related disease and injury, and these conditions have been further amplified by the COVID-19 pandemic. The SEM provides a framework for the development of complex and holistic interventions that ensures that businesses and workers are identifying and addressing their immediate health and safety needs, while also building towards policy change that will sustain positive change over time. The incorporation of tools such as the hierarchy of controls will push interventionists towards considering the implementation of existing and new public policies that will have the greatest impact on the largest number of people. While we have focused on developing this framework for workers in small businesses, this same framework could be easily adapted to other vulnerable worker populations, such as meat-packing workers and hospital cleaning crews, whose vulnerability has been highlighted by the pandemic. By incorporating frameworks rooted in public health science, government agencies, such as OSHA, could play an important role in stopping the COVID-19 pandemic through exposure reduction and applying the same principles to increase vaccine uptake. However, if government entities continue to fail at protecting workers' health, this framework also provides examples for how communities and small businesses can have agency and protect their own health.

Acknowledgements The authors acknowledge the contributions of Rachel F. Spitz for development of Fig. 3 depicting the relationship of SEM and the hierarchy of controls.

Funding This project is funded by NIH grant 5R01ES028250. This work was supported by the National Institutes of Health under Grant R01ES028250 and Grant P30ES006694. The publication's contents are solely the responsibility of the authors and do not necessarily represent the official views of the National Institutes of Health.

\section{Compliance with ethical standards}

Conflict of interest The authors declare no competing interests.

Publisher's note Springer Nature remains neutral with regard to jurisdictional claims in published maps and institutional affiliations. 


\section{References}

1. Steenland K, Burnett C, Lalich N, Ward E, Hurrell J. Dying for work: the magnitude of US mortality from selected causes of death associated with occupation. Am J Ind Med. 2003;43:461-82.

2. Leigh J. Economic burden of occupational injury and illness in the United States. Milbank Qu 2011;89:728-72.

3. Murray LR. Sick and tired of being sick and tired: scientific evidence, methods, and research implications for racial and ethnic disparities in occupational health. AJPH 2003;93:221-6.

4. Cooper D. Workers of color are far more likely to be paid povertylevel wages than white workers. Economic Policy Institute. 2018;21. https://www.epi.org/blog/workers-of-color-are-far-morelikely-to-be-paid-poverty-level-wages-than-white-workers/

5. McClure ES, Vasudevan P, Bailey Z, Patel S, Robinson WR. Racial capitalism within public health: how occupational settings drive COVID-19 disparities. Am J Epidemiol. 2020;189:1244-53.

6. Stiehl E, Shivaprakash N, Thatcher E, Ornelas IJ, Kneipp S, Baron SL, et al. Worksite promotion for low-wage workers: a scoping literature review. Am J Health Promot. 2018;32:359-73.

7. Peckham TK, Baker MG, Camp JE, Kaufman JD, Seixas NS. Creating a Future for Occupational Health. Ann Work Expo Health. 2017;81:3-15.

8. Michaels D, Wagner GR. Occupational Safety and Health Administration (OSHA) and worker safety during the COVID-19 pandemic. JAMA. 2020;324:1389-90. https://doi.org/10.1001/ja ma.2020.16343 [Online ahead of print].

9. Cherniack M, Henning R, Merchant JA, Punnett L, Sorensen GR, Wagner G. Statement on national worklife priorities. Am J Ind Med. 2011;54:10-20.

10. Sorensen G, Stoddard AM, LaMontagne AD, Emmons K, Hunt MK, Youngstrom R, et al. A comprehensive worksite cancer prevention intervention: behavior change results from a randomized controlled trial (United States). J Public Health Policy. 2003;24:5-25.

11. Baron SL, Beard S, Davis LK, Delp L, Forst L, Kidd-Taylor A, et al. Promoting integrated approaches to reducing health Inequities among low-income workers: applying a social ecological framework. Am J Ind Med. 2014;57:533-56.

12. Ahonen EQ, Fujishiro K, Cunningham T, Flynn M. Work as an inclusive part of population health inequities research and prevention. AJPH. 2018;108:306-11.

13. Xiao GB, Pan CB, Cai YZ, Lin H, Fu ZM. Effect of benzene, toluene, xylene on the semen quality and the function of accessory gonad of exposed workers. Ind Health. 2001;39:206-10.

14. LoSasso GL, Rapport LJ, Axelrod BN. Neuropsychological symptoms associated with low-level exposure to solvents and (meth) acrylates among nail technicians. Cogn Behav Neurol. 2001;14:183-9.

15. Kaukiainen A, Akila R, Martikainen R, Sainio M. Symptom screening in detection of occupational solvent-related encephalopathy. Int Arch Occup Environ Health. 2009;82:343.

16. Moulin P, Magnan A, Lehucher-Michel M-P. Occupational allergic contact dermatitis and asthma due to a single low molecular weight agent. J Occupational Health. 2008;51:91-6.

17. Chang A, Schnall AH, Law R, Bronstein AC, Marraffa JM, Spiller $\mathrm{HA}$, et al. Cleaning and Disinfectant Chemical Exposures and Temporal Associations with COVID-19-National Poison Data System, United States, January 1, 2020-March 31, 2020. MMWR. 2020;69:496.

18. Morse T, Dillon C, Weber J, Warren N, Bruneau H, Fu R. Prevalence and reporting of occupational illness by company size: population trends and regulatory implications. Am J Ind Med. 2004;45:361-70.
19. Bureau of Labor Statistics. Employer-Reported Workplace Injuries and Illnesses - 2016. Washington D.C.: U.S. Department of Labor; 2017.

20. Seabury SA, Terp S, Boden LI. Racial and ethnic differences in the frequency of workplace injuries and prevalence of workrelated disability. Health Aff. 2017;36:266-73.

21. Thornton RL, Glover CM, Cené CW, Glik DC, Henderson JA, Williams DR. Evaluating strategies for reducing health disparities by addressing the social determinants of health. Health Aff. 2016;35:1416-23.

22. Selden TM, Berdahl TA. COVID-19 and racial/ethnic disparities in health risk, employment, and household composition. Health Aff. 2020. https://doi.org/10.1377/hlthaff. 2020.00897.

23. Okun A, Lentz TJ, Schulte P, Stayner L. Identifying high-risk small business industries for occupational safety and health interventions. Am J Ind Med. 2001;39:301-11.

24. Anger WK, Stupfel J, Ammerman T, Tamulinas A, Bodner T, Rohlman DS. The suitability of computer-based training for workers with limited formal education: a case study from the US agricultural sector. Int J Train Dev. 2006;10:269-84.

25. Brunette MJ. Development of educational and training materials on safety and health: Targeting Hispanic workers in the construction industry. Fam Community Health. 2005;28:253-66.

26. Roelofs CR, Barbeau EM, Ellenbecker MJ, Moure-Eraso R. Prevention strategies in industrial hygiene: a critical literature review. AIHA J. 2003;64:62-7.

27. Brosseau LM, Bejan A, Parker DL, Skan M, Xi M. Workplace safety and health programs, practices, and conditions in auto collision repair businesses. J J Occup Environ Hyg. 2014;11:354-65.

28. Eaton LA, Kalichman SC. Social and behavioral health responses to COVID-19: lessons learned from four decades of an HIV pandemic. J Behav Med. 2020;43:341-5.

29. Golden SD, Earp JL. Social Ecological Approaches to Individuals and Their Contexts: Twenty Years of Health Education \& Behavior Health Promotion Interventions. Health Educ Behav. 2012; 39:364-72.

30. Campe J, Hoare L, Hagopian A, Keifer M. Using community-based methods and a social ecological framework to explore workplace health and safety of bloqueros on the olympic peninsula. Am J Ind Med. 2011;54:438-49.

31. Ramírez DM, Ramírez-Andreotta MD, Vea L, Estrella-Sánchez R, Wolf AMA, Kilungo A, et al. Pollution prevention through peer education: a community health worker and small and home-based business initiative on the Arizona-Sonora Border. Int $\mathrm{J}$ Environ Res Public Health. 2015;12:11209-26.

32. Azaroff LS, Nguyen HM, Do T, Gore R, Goldstein-Gelb M. Results of a community-university partnership to reduce deadly hazards in hardwood floor finishing. J Community Health. 2011;36:658-68.

33. Tucker P, Liao Y, Giles WH, Liburd L The REACH 2010 logic model: an illustration of expected performance. Prev Chron Dis 2006;3. http://www.cdc.gov/pcd/issues/2006/jan/05_0131.htm.

34. M McQuade S, Novoa M, Obernauer C Building a healthier nail salon industry. Stanford Social Innovation Review. 2016. Spring. https://ssir.org/articles/entry/building_a_healthier_nail_ salon_industry\#.

35. Office of the Governor Pressroom. Governor Cuomo announces new ventilation standards for nail salons. 2016. https://www. governor.ny.gov/news/governor-cuomo-announces-new-ventila tion-standards-nail-salons.

36. Agency for Toxic Substances and Disease Registry Environmental Toxicants and Breast Cancer. Hazard Subst Public Health. 2004;4:1-14.

37. Cozzens T. Defeating the Devil's Arm: The Victory over the ShortHandled Hoe in California Agriculture. Agric Hist 2015;89:494-512. 